Dhaka Univ. J. Sci. 60(2): 191-195, 2012 (July)

\title{
Connections on Bundles
}

\author{
Md. Showkat Ali, Md. Mirazul Islam, Farzana Nasrin, Md. Abu Hanif Sarkar and Tanzia Zerin Khan \\ Department of Mathematics, University of Dhaka, Dhaka 1000, Bangladesh, \\ Email: msa317@yahoo.com
}

Received on 25. 05. 2011.Accepted for Publication on 15. 12. 2011

\begin{abstract}
This paper is a survey of the basic theory of connection on bundles. A connection on tangent bundle $T M$, is called an affine connection on an $m$-dimensional smooth manifold $M$. By the general discussion of affine connection on vector bundles that necessarily exists on $M$ which is compatible with tensors.
\end{abstract}

\section{Introduction}

In order to differentiate sections of a vector bundle [5] or vector fields on a manifold we need to introduce a structure called the connection on a vector bundle. For example, an affine connection is a structure attached to a differentiable manifold so that we can differentiate its tensor fields. We first introduce the general theorem of connections on vector bundles. Then we study the tangent bundle. $T M$ is a $m$-dimensional vector bundle determine intrinsically by the differentiable structure [8] of an $\mathrm{m}$ dimensional smooth manifold $M$.

\section{Connections on Vector Bundles}

A connection on a fiber bundle [7] is a device that defines a notion of parallel transport on the bundle, that is, a way to connect or identify fibers over nearby points. If the fiber bundle is a vector bundle, then the notion of parallel transport is required to be linear. Such a connection is equivalently specified by a covariant derivative, which is an operator that can differentiate sections of that bundle along tangent directions in the base manifold [3]. Connections in this sense generalize, to arbitrary vector bundles, the concept of a linear connection on the tangent bundle of a smooth manifold, and are sometimes known as linear connections. Nonlinear connections are connections that are not necessarily linear in this sense.

Definition 1. A connection on a vector bundle $E$ is a map

$D: \Gamma(E) \rightarrow$

$\Gamma\left(T^{*} M \otimes E\right)$

which satisfies the following conditions:

(i) For any $s_{1}, s_{2} \in \Gamma(E)$,

$$
D\left(s_{1}+s_{2}\right)=D s_{1}+D s_{2}
$$

(ii) For $s \in \Gamma(E)$ and any $\alpha \in C^{\infty}(M)$,

$$
D(\alpha s)=d \alpha \otimes s+\alpha D s
$$

Suppose $X$ is a smooth tangent vector fields on $M$ and $s \in \Gamma(E)$. Let

$$
D_{X} s=\langle X, D s>
$$

where $<,>$ represents the pairing between $T M$ and $T^{*} M$. Then $D_{X} S$ is a section of $E$, called the absolute differential quotient or the covariant derivative of the section $s$ along $X$.

Theorem 1. A connection always exists on a vector bundle.

Proof. Choose a coordinate covering $\left\{U_{\alpha}\right\}_{\alpha \in A}$ of $M$. Since vector bundles are trivial locally, we may assume that there is local frame field $S_{\alpha}$ for any $U_{\alpha}$. By the local structure of connections, we need only construct a $q \times q$ matrix $w_{\alpha}$ on each $U_{\alpha}$ such that the matrices satisfy

$$
w^{\prime}=d A \cdot A^{-1}+A \cdot w \cdot A^{-1}
$$

under a change of the local frame field, which is the transformation formula for a connection, a most important formula in differential geometry.

We may assume that $\left\{U_{\alpha}\right\}$ is locally finite, and $\left\{g_{\alpha}\right\}$ is a corresponding sub-ordinate partition of unity such that supp $g_{\alpha} \subset U_{\alpha}$. When $U_{\alpha} \cap U_{\beta} \neq \varnothing$, there naturally exists a non-degenerate matrix $A_{\alpha \beta}$ of smooth functions on $U_{\alpha} \cap U_{\beta}$ such that

$$
S_{\alpha}=A_{\alpha \beta} \cdot S_{\beta}, \operatorname{det} A_{\alpha \beta} \neq 0
$$

For every $\alpha \in A$, choose an arbitrary $q \times q$ matrix $\phi_{\alpha}$ of differential 1-forms on $U_{\alpha}$. Let

$$
\begin{aligned}
w_{\alpha}=\sum_{\beta \in A} g_{\beta} \cdot( & d A_{\alpha \beta} \cdot A_{\alpha \beta}^{-1} \\
& \left.+A_{\alpha \beta} \cdot \phi_{\beta} \cdot A_{\alpha \beta}^{-1}\right)
\end{aligned}
$$

where the terms in the sums over $\beta$ with $U_{\alpha} \cap U_{\beta}=\varnothing$ are zero. Then $w_{\alpha}$ is a matrix of differential 1-forms on $U_{\alpha}$. We need only demonstrate the following transformation formula for $U_{\alpha} \cap U_{\beta} \neq \varnothing$ :

$$
w_{\alpha}=d A_{\alpha \beta} \cdot A_{\alpha \beta}^{-1}+A_{\alpha \beta} \cdot w_{\beta} \cdot A_{\alpha \beta}^{-1} .
$$

This can be done by a direct calculation. First observe that when $U_{\alpha} \cap U_{\beta} \cap U_{\gamma} \neq \varnothing$, the following is true in the intersection:

$$
A_{\alpha \beta} \cdot A_{\beta \gamma}=A_{\alpha \gamma} .
$$

Thus on $U_{\alpha} \cap U_{\beta} \neq \varnothing$ we have 


$$
\begin{aligned}
A_{\alpha \beta} \cdot w_{\beta} \cdot A_{\alpha \beta}^{-1}= & \sum_{\substack{\gamma \\
U_{\alpha} \cap U_{\beta} \cap U_{\gamma} \neq \emptyset\\
}} g_{\gamma} \cdot A_{\alpha \beta} \cdot\left(d A_{\beta \alpha} \cdot A_{\beta \alpha}^{-1}\right. \\
= & \left.A_{\beta \gamma} \cdot \phi_{\gamma} \cdot A_{\beta \gamma}^{-1}\right) \cdot A_{\alpha \beta}^{-1} \\
= & w_{\alpha}-d A_{\alpha \beta} \cdot A_{\alpha \beta}^{-1}
\end{aligned}
$$

This is precisely (6). We see from the above that there is much freedom in the choice of a connection. This completes the proof of the theorem.

Remark 1. In particular, if we let $\phi_{\beta}=0$ in (6), then we obtain a connection $D$ on $E$ whose connection matrix on $U_{\alpha}$ is

$$
w_{\alpha}=\sum_{\beta} g_{\beta} \cdot\left(d A_{\alpha \beta} \cdot A_{\alpha \beta}^{-1}\right)
$$

By the transformation formula (3) for connection matrices, the vanishing of a connection matrix is not an invariant property. In fact, for an arbitrary connection, we can always find a local frame field with respect to which the connection matrix is zero at some point. This fact is useful in calculations involving connections.

Theorem 2. Suppose $D$ is a connection on a vector bundle $E$, and $p \in M$. Then there exists a local frame field $S$ in a coordinate neighborhood of $p$ such that the corresponding connection matrix $w$ is zero at $p$.

Proof. Choose a coordinate neighborhood $\left(U ; u^{i}\right)$ of $p$ such that $u^{i}(p)=0,1 \leq i \leq m$. Suppose $S^{\prime}$ is a local frame field on $U$ with corresponding connection matrix $w^{i}=\left(w_{\alpha}^{\prime \beta}\right)$,

where

$$
w_{\alpha}^{\prime \beta}=\sum_{i=1}^{m} \Gamma_{\alpha i}^{\prime \beta} u^{i}
$$

and the $\Gamma_{\alpha i}^{\prime \beta}$ are smooth functions on $U$. Let

$$
a_{\alpha}^{\beta}=\delta_{\alpha}^{\beta}-\sum_{i=1}^{m} \Gamma_{\alpha i}^{\prime \beta}(p) \cdot u^{i}
$$

Then $A=\left(a_{\alpha}^{\beta}\right)$ is the identity matrix at $p$. Hence there exists a neighborhood $V \subset U$ of $p$ such that $A$ is nondegenerate in $V$. Thus

$$
S=A \cdot S^{\prime}
$$

is a local frame field on $V$. Since

$$
d A(p)=-w^{\prime}(p),
$$

we can obtain from $(3)$,

$$
\begin{aligned}
w(p) & =\left(d A \cdot A^{-1}+A \cdot w^{\prime} \cdot A^{-1}\right)(p) \\
& =-w^{\prime}(p)+w^{\prime}(p) \\
& =0
\end{aligned}
$$

Thus $S$ is the desired local frame field.

Theorem 3. Suppose $X, Y$ are two arbitrary smooth tangent vector fields on the manifold $M$. Then

$R(X, Y)=D_{X} D_{Y}-D_{Y} D_{X}-D_{[X, Y]}$

Proof. Because the absolute differential quotient and the curvature operator are local operators, we need only consider the operations of both sides of (9) on a local section. Suppose $s \in \Gamma(E)$ has the local expression

$$
s=\sum_{\alpha=1}^{q} \lambda^{\alpha} s_{\alpha}
$$

Then

$$
D_{X} s=\sum_{\alpha=1}^{q}\left(X \lambda^{\alpha}+\sum_{\beta=1}^{q} \lambda^{\beta}<X, w_{\beta}^{\alpha}>\right) s_{\alpha},
$$

and $D_{Y} D_{X} s=\sum_{\alpha=1}^{q}\left\{Y\left(X \lambda^{\alpha}\right)+\sum_{\beta=1}^{q}\left(X \lambda^{\beta}<Y, w_{\beta}^{\alpha}>\right.\right.$

$$
\left.+Y \lambda^{\beta}<X, w_{\beta}^{\alpha}>\right)
$$

$\left.\sum_{\beta=1}^{q} \lambda^{\beta}\left(Y<X, w_{\beta}^{\alpha}>+\sum_{\gamma=1}^{q}<X, w_{\beta}^{\gamma}><Y, w_{\gamma}^{\alpha}>\right)\right\} s_{\alpha}$.

Hence $\quad D_{X} D_{Y} s-D_{Y} D_{X} s=\sum_{\alpha=1}^{q}\left\{[X, Y] \lambda^{\alpha}+\sum_{\beta=1}^{q} \lambda^{\beta}(<\right.$ $\left.\left.[X, Y], w_{\beta}^{\alpha}>+<X \Lambda Y, d w_{\beta}^{\alpha}>-\sum_{\gamma=1}^{q} w_{\beta}^{\gamma} \Lambda w_{\gamma}^{\alpha}>\right)\right\} s_{\alpha}=$ $D_{[X, Y]} S+\sum_{\alpha, \beta=1}^{q} \lambda^{\beta}<X \Lambda Y, \Omega_{\beta}^{\alpha}>s_{\alpha}$

That is,

$$
R(X, Y) s=D_{X} D_{Y} s-D_{Y} D_{X} s-D_{[X, Y]} s
$$

This completes the proof of the theorem.

Theorem 4. The curvature matrix $\Omega$ satisfies the Bianchi identity

$$
d \Omega=w \Lambda \Omega-\Omega \Lambda w .
$$

Proof: Apply exterior differentiation [9] to both sides of $\Omega=d w-w \Lambda w d \Omega=-d w \Lambda w+w \Lambda d w$

$$
\begin{aligned}
& =-(\Omega+w \Lambda w) \Lambda w+w \Lambda(\Omega+w \Lambda w) \\
& =w \Lambda \Omega-\Omega \Lambda w
\end{aligned}
$$

This completes the proof of the theorem.

Remark 2. If a section $s$ of a vector bundle $E$ satisfies the condition $D s=0$, then $s$ is called a parallel section.

\section{Affine Connections}

Definition 2. Let $M$ be a smooth n-dimensional manifold, $O_{M}$ be the set of smooth functions and $\Gamma(T M)$ be the vector space of smooth vector fields. An affine connection on $M$ is a map (denoted by $\nabla$ )

$$
\begin{aligned}
\nabla: \Gamma(T M) \times \Gamma(T M) & \rightarrow \Gamma(T M) \\
(X, Y) & \mapsto \nabla_{X} Y
\end{aligned}
$$

such that

(i) $\nabla_{X}\left(Y_{1}+Y_{2}\right)=\nabla_{X} Y_{1}+\nabla_{X} Y_{2}$

(ii) $\nabla_{X_{1}+X_{2}} Y=\nabla_{X_{1}} Y+\nabla_{X_{2}} Y$ 


$$
\begin{aligned}
& \text { (iii) } \nabla_{X}(f Y)=X(f) Y+f \nabla_{X} Y \\
& \text { (iv) } \nabla_{f X} Y=f \nabla_{X} Y ; \forall f \in O_{M} \text { and } \\
& X, Y \in \Gamma(T M)
\end{aligned}
$$

\section{Affine Connection in Two Coordinates Charts}

Let $(U, \varphi)$ be a coordinate chart on a manifold $M$, with coordinates $\left(x^{1}, x^{2}, \ldots, x^{n}\right)$. Then the vector fields $X$ and $Y$ can be expressed as

$$
\begin{aligned}
& X=\sum_{i=1}^{n} X^{i}(x) \frac{\partial}{\partial x^{i}} \\
& Y=\sum_{j=1}^{n} Y^{j}(x) \frac{\partial}{\partial x^{j}}
\end{aligned}
$$

For some smooth functions $X^{i}(x)$ and $Y^{j}(x)$. In $U, \frac{\partial}{\partial x^{i}}$ are smooth vector fields. $\nabla_{\frac{\partial}{\partial x^{i}}} \frac{\partial}{\partial x^{j}}$ is again a smooth vector field. Thus

$$
\nabla_{\frac{\partial}{\partial x^{i}}} \frac{\partial}{\partial x^{j}}=\sum_{k=1}^{n} \Gamma_{i j}^{k} \frac{\partial}{\partial x^{k}}
$$

For some smooth functions $\Gamma_{i j}^{k}(x)$. Here $\Gamma_{i j}^{k}(x)$ is a $n^{3}$ function.

$$
\begin{gathered}
\Rightarrow \nabla_{e_{i}} e_{j}=\sum_{k=1}^{n} \Gamma_{i j}^{k} e_{k} ; \text { where } e_{i}=\frac{\partial}{\partial x^{i}}, e_{j}=\frac{\partial}{\partial x^{j}} \\
\text { and } e_{k}=\frac{\partial}{\partial x^{k}}
\end{gathered}
$$

Let us compute $\nabla_{X} Y$

$$
\nabla_{X} Y=\nabla_{\sum_{i=1}^{n} X^{i} e_{i}} \sum_{j=1}^{n} Y^{j} e_{j}
$$

$=\sum_{j=1}^{n}\left(\nabla_{\sum_{i=1}^{n} X^{i} e_{i}} Y^{j} e_{j}\right)$

[By $\operatorname{axiom}(i)]$

$=\sum_{i=1}^{n} \sum_{j=1}^{n}\left(\nabla_{X^{i} e_{i}} Y^{j} e_{j}\right)$

[By axiom $(i i)]$

$=\sum_{i=1}^{n} \sum_{j=1}^{n}\left(X^{i} \nabla_{e_{i}} Y^{j} e_{j}\right)$

[By $\operatorname{axiom}(i v)]$

$=\sum_{i=1}^{n} \sum_{j=1}^{n} X^{i}\left(e_{i}\left(Y^{j}\right) e_{j}+Y^{j} \nabla_{e_{i}} e_{j}\right)$ [By axiom $\left.(i i i)\right]$

$$
\nabla_{X} Y=\sum_{i=1}^{n} \sum_{j=1}^{n} X^{i}\left(\frac{\partial}{\partial x^{i}}\left(Y^{j}\right) e_{j}+\sum_{k=1}^{n} \Gamma_{i j}^{k} e_{k} Y^{j}\right)
$$

The functions $\Gamma_{i j}^{k}(x)$ are called coordinate symbols of the affine connection $\nabla$. The vector field $\nabla_{X} Y$ is often called covariant derivative of vector field $Y$ along the vector field $X$.

Definition 3. If the torsion tensor of an affine connection $\nabla$ is zero, then the connection is said to be torsion free.

A torsion-free affine connection always exists. In fact, if the coefficients of a connection $\nabla$ are $\Gamma_{j k}^{i}$, then the set

$$
\tilde{\Gamma}_{j k}^{i}=\frac{1}{2}\left(\Gamma_{i k}^{j}+\Gamma_{k i}^{j}\right) \text {. }
$$

Obviously, $\tilde{\Gamma}_{j k}^{i}$ is symmetric with respect to the lower indices and satisfies

$$
\Gamma_{i k}^{\prime j}={ }_{p r}^{q} \frac{\partial w^{j}}{\partial u^{q}} \frac{\partial u^{p}}{\partial w^{i}} \frac{\partial u^{r}}{\partial w^{k}}+\frac{\partial^{2} u^{p}}{\partial w^{i} \partial w^{k}} \cdot \frac{\partial w^{j}}{\partial u^{p}}
$$

under a local change of coordinates. Therefore the $\tilde{\Gamma}_{i k}^{j}$ are the coefficients of some connection $\tilde{\nabla}$ and $\tilde{\nabla}$ is torsion-free.

Theorem 5. Suppose $\nabla$ is a torsion-free affine connection on $M$. Then for any point $p \in M$ there exists a local coordinate system $u^{i}$ such that the corresponding connection coefficients $\Gamma_{i k}^{j}$ vanish at $p$.

Proof. Suppose $\left(W ; w^{i}\right)$ is a local coordinating system at $p$ with connection coefficients $\tilde{\Gamma}_{i k}^{\prime j}$. Let $u^{i}=w^{i}+\frac{1}{2} \Gamma_{j k}^{i}(p)\left(w^{j}-w^{j}(p)\right)\left(w^{k}\right.$

$$
\left.-w^{k}(p)\right)
$$

Then, $\left.\frac{\partial u^{i}}{\partial w^{j}}\right|_{p}=\delta_{j}^{i},\left.\quad \frac{\partial^{2} u^{i}}{\partial w^{i} \partial w^{k}}\right|_{p}=\Gamma_{j k}^{\prime i}(p)$

Thus the matrix $\left(\frac{\partial u^{i}}{\partial w^{j}}\right)$ is non-degenerate near $p$, and (13) provides for a change of local coordinates in a neighborhood of $p$. From (12) we see that the connection coefficients $\Gamma_{i k}^{j}$ in the new coordinate system $u^{i}$ satisfy

$$
\Gamma_{i k}^{j}(p)=0 \quad ; \quad 1 \leq i, j, k \leq m
$$

This completes the proof of the theorem.

Theorem 6. Suppose $\nabla$ is a torsion-free affine connection on $M$. Then we have the Bianchi identity:

$$
R_{i k l, h}^{j}+R_{i l h, k}^{j}+R_{i h k, l}^{j}=0 .
$$

Proof. From Theorem 4, we have

$$
d \Omega_{i}^{j}=w_{i}^{k} \Lambda \Omega_{k}^{j}-\Omega_{i}^{k} \Lambda w_{k}^{j},
$$

that is,

$$
\begin{aligned}
& \frac{\partial R_{i k l}^{j}}{\partial u^{h}} d u^{h} \Lambda d u^{k} \Lambda d u^{l} \\
&=\left(\Gamma_{i h}^{p} R_{p k l}^{j}-\Gamma_{p h}^{j} R_{i k l}^{p}\right) d u^{h} \Lambda d u^{k} \Lambda d u^{l}
\end{aligned}
$$

Therefore

$R_{i k l, h}^{j} d u^{h} \Lambda d u^{k} \Lambda d u^{l}=$ $-\left(\Gamma_{k h}^{p} R_{i p l}^{j}-\Gamma_{l h}^{p} R_{i k p}^{j}\right) d u^{h} \Lambda d u^{k} \Lambda d u^{l}=0$,

where in the last equality we have used the torsion-free property of the connection. Hence

$$
\left(R_{i k l, h}^{j}+R_{i l h, k}^{j}+R_{i h k, l}^{j}\right) d u^{h} \Lambda d u^{k} \Lambda d u^{l}=0
$$

Now since the coefficients of (15) are skew-symmetric with respect to $k, l, h$, we have

$$
R_{i k l, h}^{j}+R_{i l h, k}^{j}+R_{i h k, l}^{j}=0
$$

This completes the proof of the theorem. 


\section{Connection Compatible with Tensors}

Let $M$ be a smooth manifold and $\tau$ be any tensor in $M$. Mostly this can be interested in the case when $\tau=g$ is a semi-Riemannian metric tensor on $M$, i.e., $\tau$ is a non-degenerate [1] symmetric (2,0)- tensor, or when $\tau=\omega$ is symplectic form on $M$, i.e., $\tau$ is a non-degenerate closed 2-form [7], [9]. If $\nabla$ is a connection in $M$, i.e., a connection on the tangent bundle $T M$, then we have naturally induced connections on all tensor bundles on $M$, all of which is denoted by the same symbol $\nabla$.

Definition 4. The torsion of $\nabla$ is the anti-symmetric tensor

$$
T(X, Y)=\nabla_{X} Y-\nabla_{Y} X-[X, Y],
$$

where $[X, Y]$ denotes the Lie brackets of the vector fields $X$ and $Y ; \nabla$ is called symmetric if $T=0$.

The connection $\nabla$ is said to be compatible with $\tau$ is $\nabla$ - parallel, i.e., when $\nabla \tau=0$.

Establishing whether a given tensor $\tau$ admits compatible connections is a local problem. Namely, one can use partition of unity to extend locally defined connections and observe that a convex combination of compatible connections is a compatible connection. In local coordinates, finding a connection compatible with a given tensor reduces to determining the existence of solutions for a non homogeneous linear system for the Christoffel symbols of the connection.

It is well known that semi-Riemannian metric tensors admit a unique compatible symmetric connection, called the Levi-Civita connection of the metric tensor, which can be given explicitly in [4]. Uniqueness of the Levi-Civita connection can be obtained by a curious combinatorial argument, as follows.

Suppose that $\nabla$ and $\tilde{\nabla}$ are connections on $M$; their difference $\nabla-\tilde{\nabla}$ is a tensor, that is denoted by $t$

$$
t(X, Y)=\tilde{\nabla}_{X} Y-\nabla_{X} Y,
$$

where $X$ and $Y$ are smooth vector fields on $M$. If both $\nabla$ and $\tilde{\nabla}$ are symmetric connection, then $t$ is symmetric

$$
\begin{aligned}
& t(X, Y)-t(Y, X)=\tilde{\nabla}_{X} Y-\nabla_{X} Y-\tilde{\nabla}_{Y} X+\nabla_{Y} X \\
& =[X, Y]+[Y, X]=0 .
\end{aligned}
$$

Lemma 1. Let $U$ be a set and $\rho: U \times U \times U \rightarrow \nabla$ be a map that is symmetric in its first two variables and anti-symmetric in its last two variables. Then $\rho$ is identically zero.

Proof. Let $u_{1}, u_{2}, u_{3} \in U$ be fixed. We have $\rho\left(u_{1}, u_{2}, u_{3}\right)=\rho\left(u_{2}, u_{1}, u_{3}\right)=-\rho\left(u_{2}, u_{3}, u_{1}\right)=$

$-\rho\left(u_{3}, u_{2}, u_{1}\right)$,

so that $\rho$ is anti-symmetric in the first and the third variables. On the other hand

$\rho\left(u_{1}, u_{2}, u_{3}\right)=-\rho\left(u_{3}, u_{2}, u_{1}\right)=-\rho\left(u_{2}, u_{3}, u_{1}\right)$

$=\rho\left(u_{1}, u_{3}, u_{2}\right)$,

so that $\rho$ is symmetric in the second and the third variables. This concludes the proof.

Theorem 7. There exists at most one symmetric connection which is compatible with a semi Riemannian metric.

Proof. Assume that $\mathrm{g}$ is a semi-Riemannian metric on $M$, and let $\nabla$ and $\tilde{\nabla}$ are two symmetric connections such that $\nabla g=\tilde{\nabla} g=0 ; \quad$ for all $\quad p \in M, \quad$ consider the map $\rho: T_{p} M \times T_{p} M \times T_{p} M \rightarrow \nabla$ given by

$$
\rho(X, Y, Z)=g(t(X, Y), Z),
$$

where $t$ is the difference $\nabla-\tilde{\nabla}$. Since $t$ is symmetric, then $\rho$ is symmetric in the first two variables. On the other hand, $\rho$ is anti-symmetric in the last two variables

$$
\begin{aligned}
& \rho(X, Y, Z)+\rho(X, Z, Y)=g\left(\tilde{\nabla}_{X} Y, Z\right)- \\
& g\left(\nabla_{X} Y, Z\right)+g\left(\tilde{\nabla}_{X} Z, Y\right)-g\left(\nabla_{X} Z, Y\right) \\
& =\tilde{\nabla}_{g}(X, Y, Z)-\nabla_{g}(X, Y, Z)=0 .
\end{aligned}
$$

By Lemma $1, \rho=0$, hence $t=0$, and thus $\tilde{\nabla}=\nabla$. Hence completes the proof.

1. Arnold's V.I., 1978. Mathematical Methods of Classical Mechanics, Springer Verlag.

2. Brickell, F. and Clark, R.S., $1970 . \quad$ Differential Manifolds: An Introduction, Van Nostrand Reinhold Company, London.

3. Carno, M.P. do, 1992, Riemannian geometry, Birkh auser, Boston.

4. Chern, S.S. Chern, W.H., K.S., Law, Lectures on Differential Geometry.

5. Gupta, P.P. and Malik, G.S. 2000. Tensors and Differential Geometry, Pragati Prakashan, Meerut, U.P., India.

6. Islam, J. Chrish, 1989. Modern Differential Geometry for Physicists, World Scientific Publishing Co. Pte. Ltd.

7. Kobayashi, S. and Nomizu, K., 1996. Foundations of Differential Geometry, Volume 1, John Wiley and Sons, Interscience, New York.

8. Novikov, S.P. and Fomenko. A.T., Basic Elements of Differential Geometry and Topology.

9. Struik, D.J., 1950. Lectures on Classical Differential Geometry, Addition-Weslely Publishing Co., Inc. 